\title{
Study of the parsec-scale jet in the blazar 3C 66A with VLBA
}

\author{
Guang-Yao Zhao ${ }^{1,2,3}$ Yong-Jun Chen ${ }^{1,3}$ Zhi-Qiang Shen ${ }^{1,3}$ \\ Hiroshi Sudou ${ }^{4}$ Satoru Iguchi ${ }^{5}$ Yasuhiro Murata $^{6}$ Yoshiaki Taniguchi $^{7}$ \\ ${ }^{1}$ Shanghai Astronomical Observatory, Chinese Academy of Sciences,Shanghai 200030, China \\ email: gyzhao@shao.ac.cn \\ ${ }^{2}$ University of Chinese Academy of Sciences, Beijing 100049, China \\ ${ }^{3}$ Key Laboratory of Radio Astronomy, Chinese Academy of Sciences, China \\ ${ }^{4}$ Faculty of Engineering, Gifu University, Gifu 501-1193, Japan \\ ${ }^{5}$ National Astronomical Observatory of Japan, Tokyo 181-8588, Japan \\ ${ }^{6}$ The Institute of Space and Astronautical Science, JAXA, Kanagawa 229-8510, Japan \\ ${ }^{7}$ Research Center for Space and Cosmic Evolution, Ehime University, Matsuyama 790-8577, \\ Japan
}

\begin{abstract}
We report 4-epoch VLBA observations of 3C 66A at 22GHz. The resulting images show a typical core-jet structure. We combine our results with some previous results to investigate the proper motions of the jet components. The kinematics of $3 \mathrm{C} 66 \mathrm{~A}$ is quite complicated; mildly superluminal motions as well as apparent inward motions have been detected for some components. The inward motions may imply position change of the observed core.
\end{abstract}

Keywords. Radio continuum: galaxies - galaxies: individual (3C 66A) - galaxies: jet

\section{Introduction}

3C 66A (B0219+428, NRAO 102, 4C +42.07) is a well-known blazar which is further sub-classified as an intermediate synchrotron peaked BL Lac object (IBL) in terms of its SED (Abdo et al. 2010). At radio wavelengths, previous Very Long Baseline Interferometry (VLBI) images from 2.3 to $43 \mathrm{GHz}$ revealed a typical core-jet structure on parsec scales (e.g. Jorstad et al. 2001 (hereafter J01), Marscher et al. 2002, Cai et al. 2007 (hereafter C07)). The kinematics of 3C 66A jet has been studied via multi-epoch VLBI observations (e.g. Jorstad et al. 2005 (hereafter J05), Britzen et al. 2008) and superluminal motions as well as apparent inward motions have been reported. Here we re-visit these properties over a relatively longer period with the VLBA at $22 \mathrm{GHz}$. In this work, we adopt a redshift of $\mathrm{z}=0.444$ for $3 \mathrm{C} 66 \mathrm{~A}$. With $H_{0}=71 \mathrm{~km} \mathrm{~s}^{-1} \mathrm{Mpc}^{-1}$, $\Omega_{M}=0.27$, and $\Omega_{\Lambda}=0.73$, we have 1 mas $=5.69 \mathrm{pc}$.

\section{Observations and Data Reduction}

3C $66 \mathrm{~A}$ was observed as primary phase-reference calibrator for the VLBA project BS144 (Sudou \& Iguchi. 2011). $22 \mathrm{GHz}$ observations were successfully carried out at all 4 epochs $(2004.80,2005.05,2005.35,2005.54)$. The total on-source time of 3C $66 \mathrm{~A}$ at each epoch is about $40 \mathrm{~min}$. The data were recorded in VLBA format with 4 Intermediate Frequency (IF) bands, $8 \mathrm{MHz}$ each. Data correlation was done with the VLBA correlator in Socorro. The data reduction was performed with the NRAO AIPS and Caltech DIFMAP packages. Amplitude and phase calibrations were made in a standard way given in AIPS COOKBOOK, with residual phase delays and delay rates calibrated using global fringe-fitting. Bandpass calibrations were performed using $0133+476$ for all epochs. The output data were then read into DIFMAP for hybrid mapping and model fitting. Circular Gaussian models were used in model fitting. 

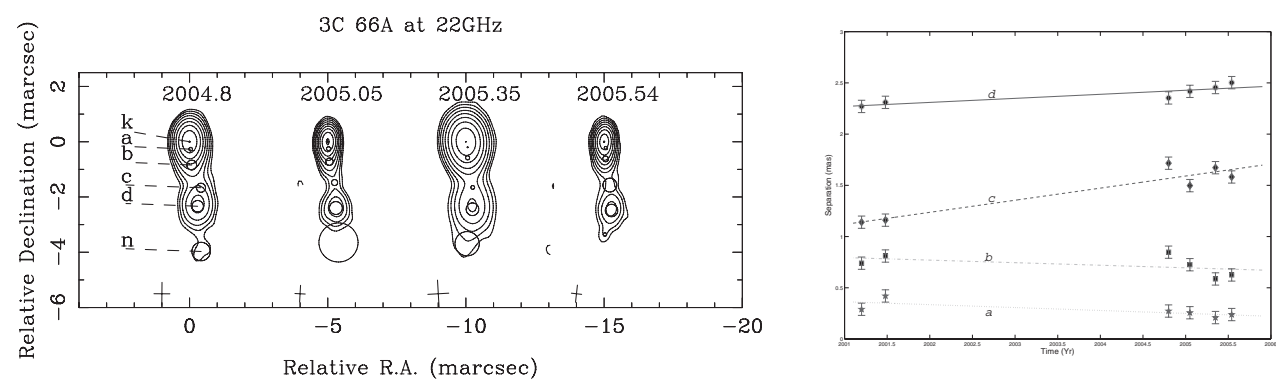

Figure 1. Left: The naturally-weighted maps of 3C 66A at $22 \mathrm{GHz}$. The crosses represent the restoring beams; Right: Proper motions of the jet components at $22 \mathrm{GHz}$. The apparent speed of each component: $a:-0.76 c, b:-0.67 c, c: 3.16 c, d: 1.06 c$.

\section{Results and Discussion}

Our VLBI maps of 3C 66A (see Fig. 1 left) show a typical core-jet structure which is similar to previous results (e.g. J01, J05, C07). The northmost component is identified as the core because it is the brightest and the most compact. Multi-frequency results show that it has a relatively flat spectrum (Zhao et al. in preparation). The jet is a smooth continuous jet roughly towards the south. The jet bends at about 1.2 mas. According to J05, the bending is continuous from 1.2 to 3 mas which might be due to the decrease of the viewing angle leading to increase of the Doppler boosting effect. The flux densities of components $k$ - $d$ in our maps support such an argument.

We combine our results with that in C07 so we could study the kinematics of the jet over a much longer time-interval, from 2001.20 to 2005.54 (see Fig. 1 right). The jet components show very complex proper motions, indicating the complex nature of this blazar. The inward motions detected for components $a$ and $b$ may be attributed to the position change of the observed core. This may be due to opacity change or the motions of the inner-most jet components which cannot be separated from the core under current resolutions. Multi-frequency studies show the core shift may change in this source, which could be related to the possible core position change in this source (Gao et al. in preparation). Components $c$ and $d$ show mildly superluminal motions. More detailed multi-frequency study of the morphology and kinematics of $3 \mathrm{C} 66 \mathrm{~A}$ can be found in Zhao et al. (in preparation).

\section{Acknowledgements}

This work is partly supported by China Ministry of Science and Technology under State Key Development Program for Basic Research (2012CB821800), the Natural Science Foundation of China (grants 10625314, 11121062, 11173046, 11273042, 12ZR1436100 and U1231106) and the CAS/SAFEA International Partnership Program for Creative Research Teams.

\section{References}

Abdo A. A., Ackermann M., Agudo I. et al. 2010, ApJ, 716, 30

Britzen S., Vermeulen R. C., Campbell R. M. et al. 2008, A\& A, 484, 119

Cai H.-B., Shen Z.-Q., Sudou H. et al. 2007, A\&A, 468, 963 (C07)

Jorstad S. G., Marscher A. P., Mattox J. R. et al. 2001, ApJS, 134, 181 (J01)

Jorstad S. G., Marscher A. P., Lister M. L., et al. 2005, AJ, 130, 1418 (J05)

Marscher A. P., Jorstad S. G., Mattox J. R., Wehrle A. E. 2002, ApJ, 577, 85

Sudou H., Iguchi S. 2011, AJ, 142, 49 